\title{
WIDE RANGE DIELECTRIC SPECTROSCOPY ON GLASS-FORMING MATERIALS: AN EXPERIMENTAL OVERVIEW
}

\author{
U. SCHNEIDER, P. LUNKENHEIMER, A. PIMENOV, \\ R. BRAND, A. LOIDL \\ Experimentalphysik V, Universität Augsburg, D-86135 Augsburg, \\ Germany
}

\begin{abstract}
Dielectric spectroscopy is one of the most commonly used techniques for the investigation of the dynamic response of glassforming materials. The tremendous slow-down of the particle motions when approaching the glass transition and especially the fast processes in glass-forming materials, which have come into the focus of scientific interest recently, make the investigation in a wide frequency range highly desirable. Recently, results from broadband dielectric spectroscopy on glass-forming materials in their liquid and supercooled-liquid state, covering more than 18 decades of frequency have been reported by our group. In the present paper, we give an overview of the various experimental setups and techniques used to collect these spectra. As an example, spectra of the prototypical glass former glycerol are presented.
\end{abstract}

Keywords broadband dielectric spectroscopy, glassforming liquids, glasses

\section{INTRODUCTION}

Despite a long history of research, the theoretical explanation of the glass transition is still one of the most challenging tasks of modern 


\section{U. SCHNEIDER et al.}

condensed matter physics. In recent years, a variety of novel approaches to the glass transition, both theoretical and phenomenological (e.g. [1, 2]), stimulated new experimental investigations especially of the high-frequency dynamics of glass-forming liquids (e.g., [3-12]). Among these, the mode coupling theory (MCT) [1] is currently most controversially discussed, explaining the glass transition in terms of a dynamic phase transition at a critical temperature $T_{c}$ significantly above the glass temperature $T_{g}$. For frequencies in the $\mathrm{GHz}-\mathrm{THz}$ region, excess contributions are predicted for the imaginary part of a generalized susceptibility, which up to now were mainly investigated by neutron and light scattering experiments [ 4 . Only recently, by combining various techniques, our group was able to obtain continuous dielectric spectra on glass-forming materials extending well into the relevant region [7-12]. For glycerol and propylene carbonate, spectra covering 18 decades of frequency and extending well into the $\mathrm{THz}$ range were obtained [10 12]. In the present paper we give a detailed description of the techniques employed to obtain these data. Typical broadband spectra, obtained by the combination of these techniques, are shown for the prototypical glass-former glycerol.

\section{$\underline{2}$ EXPERIMENTAL DETAILS}

Figure 11 gives an overview of the different techniques used in our laboratory. An extraordinary wide range of frequencies, continuously covering nearly 21 decades from $10^{-6}$ to $10^{15} \mathrm{~Hz}$, can be accessed. For cooling and heating of the samples various cryostats and ovens are used. The samples have to be supercooled with a sufficiently large cooling rate and polished sample cells have to be used to avoid crystallization. In case of an enhanced crystallization tendency it is necessary to cool directly from a temperature above the melting point to the measurement temperature.

To cover the complete frequency range, a single curve of real or imaginary part of the dielectric permittivity, $\varepsilon^{*}=\varepsilon^{\prime}-i \varepsilon^{\prime \prime}$, at a given temperature is composed using results from different setups. For some techniques the absolute values of $\varepsilon^{\prime}$ and $\varepsilon^{\prime \prime}$ are not always well defined. Possible sources of error are, e.g., stray capacitances, illdefined sample geometries or an incomplete filling of the capacitors and sample holders. In most cases the frequency ranges of the different devices overlap. This facilitates the matching of the curves 


\section{WIDE RANGE DIELECTRIC SPECTROSCOPY...}

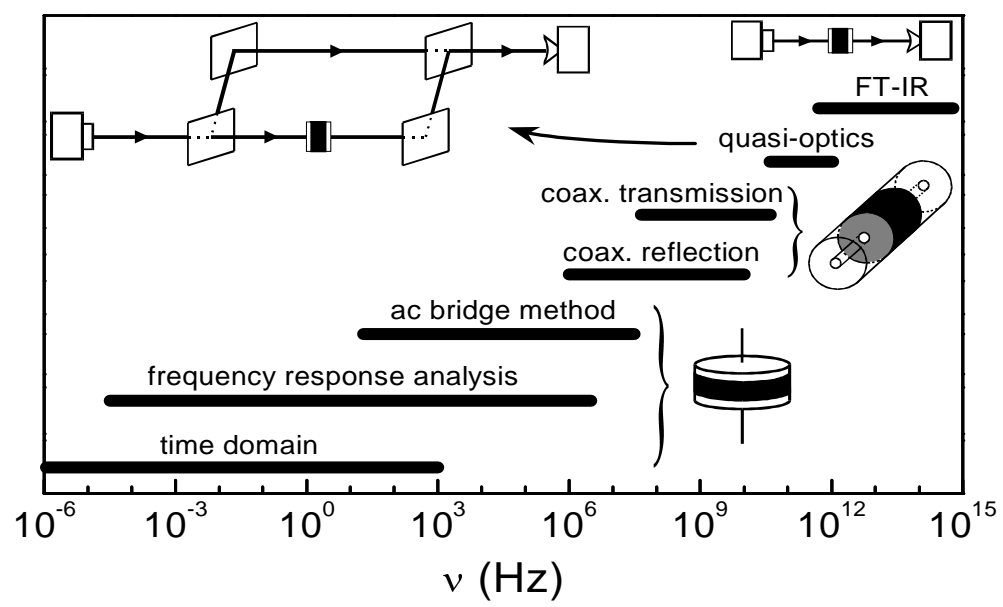

FIGURE 1: Overview of the employed techniques and corresponding frequency ranges. The sample geometries and set-ups of the spectrometers are schematically indicated in the figure.

by the application of scaling factors. In general, only one scaling factor per measurement series (with one sample, sample holder, calibration, etc.) should be applied. Overall, a sufficient number of measurements, overlapping in frequency and temperature, has to be collected to minimize the errors in the composition of broadband dielectric spectra.

\subsection{Low frequency techniques}

In the low frequency range capacitance and conductance of the sample are measured directly. The material is filled into a stainless steel capacitor of known geometry: the design consists of two circular plates kept at a distance with glass-fiber spacers or an outer support ring (see icon in Figure 1). For the measurement of low losses, large areas of the plates and small distances between them are desirable to enhance the measurement resolution. With polished surfaces and glass fibers of a few $10 \mu \mathrm{m}$ in diameter, geometric capacitances of some $100 \mathrm{pF}$ can be reached.

The lowest frequencies $(1 \mu \mathrm{Hz}<\nu<1 \mathrm{kHz})$ are reached with a home made time-domain spectrometer (TDS) [13]. The timedependent charging and discharging of the sample capacitor is measured after applying a short voltage pulse or step. Laplace transformation of the recorded response leads to frequency-dependent spectra. In the setup a Keithley electrometer measures a voltage that 


\section{U. SCHNEIDER et al.}

is proportional to the charge of the sample capacitor. Alternatively an amplifier is used, the output of which is recorded directly by an $\mathrm{AD}$-converter in a computer.

In the frequency region of $30 \mu \mathrm{Hz}<\nu<3 \mathrm{MHz}$ the so-called frequency response analysis is applied, measuring the voltage and current through the sample using lock-in techniques. In our laboratory an impedance analyser Solartron Schlumberger SI1260 in conjunction with a Chelsea Dielectric Interface and a Novovontrol alpha-analyzer are available. At higher frequencies, $10 \mathrm{~Hz}<\nu<30 \mathrm{MHz}$, the standard ac-bridge method is employed using the Hewlett-Packard LCRmeters HP4284A and HP4285A.

\section{$\underline{2.2 \text { Coaxial method }}$}

In the frequency range from a few $\mathrm{MHz}$ to about $10 \mathrm{GHz}$ a coaxial reflectometric method is used [14]. Here the sample is placed at the end of a coaxial line bridging inner and outer conductor. Either the complex reflection coefficient is detected or a direct current-voltage measurement is performed. To correct for the contributions of the coaxial line and connectors, a proper calibration using at least three standard impedances is necessary. In our laboratory the Hewlett Packard impedance analyzers HP4191A $(\nu<1 \mathrm{GHz})$ and HP4291A $(\nu<1.8 \mathrm{GHz})$ and the network analyzer HP8510B $(\nu<40 \mathrm{GHz})$ are used. The sample can be prepared as a parallel plate capacitor as depicted in Figure 2a. Inherent to this geometry are stray capaci-
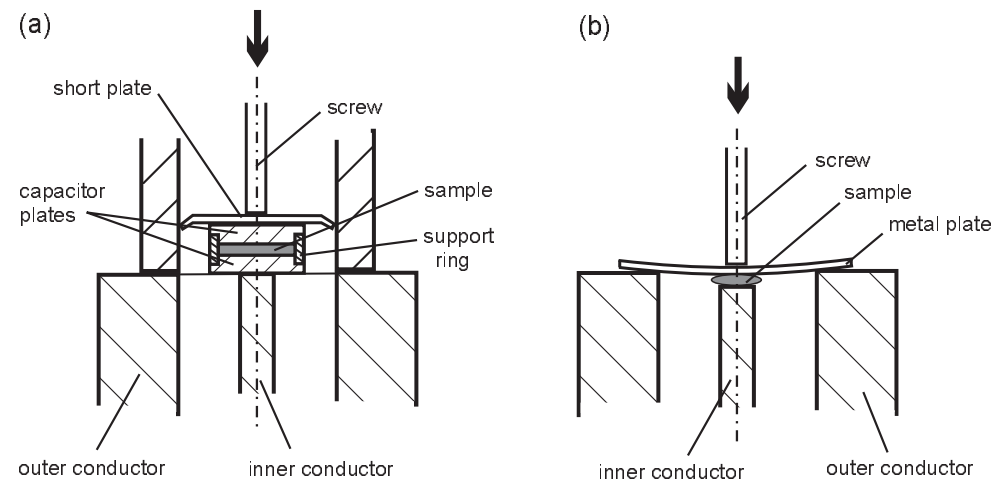

FIGURE 2: Schematic drawing of the sample geometry for reflection measurements using the parallel plate capacitor (a) and the droplet method (b). 


\section{WIDE RANGE DIELECTRIC SPECTROSCOPY...}

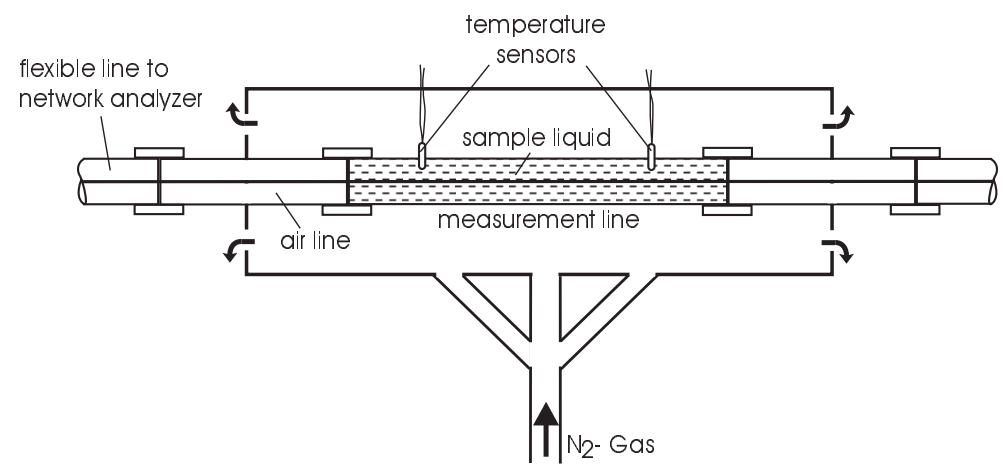

FIGURE 3: Schematic view of the setup for temperature-dependent coaxial transmission measurements.

tances and a considerable influence of the inductance of the sample holder. These drawbacks can be avoided by applying a tiny piece or droplet of the sample to the top of the inner conductor, which is somewhat shorter than the outer conductor (see Figure 20 b). By this setup losses down to $10^{-2}$ at frequencies up to $20 \mathrm{GHz}$ can be resolved, however with somewhat ill-defined absolute values only.

For higher frequencies, about $100 \mathrm{MHz}-40 \mathrm{GHz}$, the coaxial transmission method (Figure 3) can be applied [15]. The sample material fills the space between inner and outer conductor of a coaxial line which is connected to the two ports of the network analyzer HP8510B with flexible extension lines. From the measured complex transmission coefficient the dielectric permittivity of the sample material can be calculated after a proper calibration has been done. For this purpose a reflection measurement of three standards ("open", "short" and $50 \Omega$ ) at the end of the extension lines and a transmission measurement ("thru") with both ends of the extension lines connected directly is performed. For long lines the use of the empty sample line for the "thru" calibration is advantageous to correct for the signal-damping caused by line imperfections. The transmission depends exponentially on the dielectric loss. Therefore, for different temperature regions lines of various lengths between 10 and $300 \mathrm{~mm}$ have to be used to keep the transmission within the resolution window of the network analyzer. To ensure a homogeneous cooling/heating of the whole line, specially designed $\mathrm{N}_{2}$-gas cryostats have been developed (Figure 3). Stainless-steel air lines are inserted between the flexible and the measuring lines to ensure thermal decoupling. 


\section{U. SCHNEIDER et al.}

\subsection{Quasi-optical spectrometer}

The especially important frequency region $\mathrm{GHz}$ - $\mathrm{THz}$ (see introduction) can partly be covered by coaxial methods and far-infrared spectroscopy, but between some 10 and some $100 \mathrm{GHz}$ measurements are difficult with these techniques. In this region resonant cavity systems can be used, but with one cavity, only a single frequency can be investigated. An alternative is given by the free space technique where the electromagnetic wave, generated by a monochromatic source, propagates through "free space" (i.e. is unguided) and is detected by a suitable detector after passing (or being reflected by) the sample. In principle, setups as known from optical spectrometers can be applied. In our laboratory, a spectrometer developed in the group of A.A. Volkov [16, 17] is used (Figure 4). Its quasi-optical setup is that of a Mach-Zehnder interferometer. It allows to measure the frequency dependence of both, the transmission and the phase shift of a monochromatic electromagnetic beam through the sample. A frequency range $40 \mathrm{GHz}$ to $1.2 \mathrm{THz}$ is covered continuously by a set of so-called backward-wave oscillators (BWOs) which emit a monochromatic electromagnetic wave, tunable over a limited frequency range (typically by a factor of 2 ).

For the measurement the sample is put into specially designed cells made of polished stainless steel with thin plane-parallel quartz windows. Depending on the range of frequency and temperature, the thickness of the sample cell is chosen between $1 \mathrm{~mm}$ and $30 \mathrm{~mm}$ to ensure a transmission within the resolution limits of the spectrometer. The beam coming from the source is parallelized with a Teflon

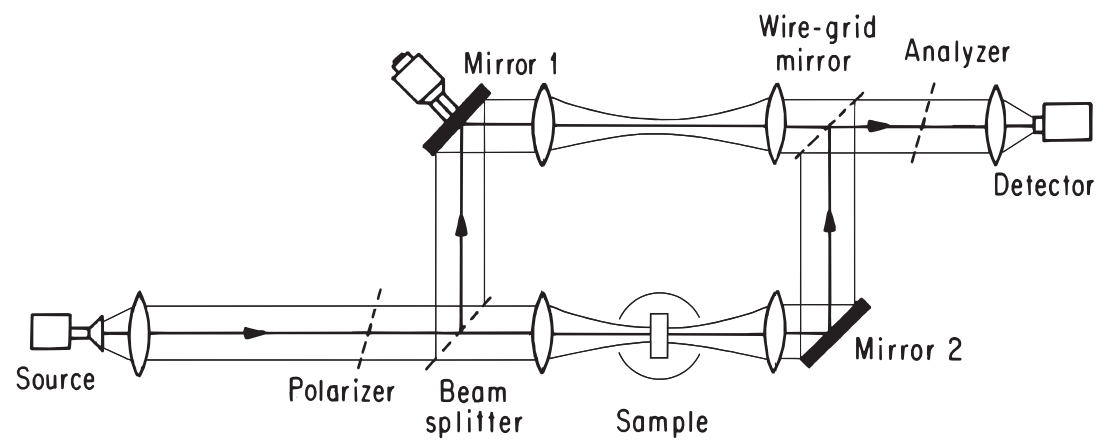

FIGURE 4: Schematic view of the setup of the quasi-optical spectrometer; the outer thin lines denote the approximate boundaries of the beam. 


\section{WIDE RANGE DIELECTRIC SPECTROSCOPY...}

or polyethylene lens. For the measurement of the transmission coefficient, only the front arm of the spectrometer is used where a set of two lenses creates a focus at the position of the sample in the cryostat or oven. The radiation is detected by a Golay cell or a pumped He-bolometer. The noise level is reduced by the use of a chopper in the beam path and lock-in techniques for detection. Two consecutive frequency sweeps with and without the sample are made at each temperature leading to a precise determination of the transmission coefficient $T$. Values down to about $T=10^{-7}$ can be detected in this way. For the determination of the phase shift, after passing a polarizer the beam is split into two with a $45^{\circ}$ wire-grid. In the second (reference) arm of the spectrometer a second set of lenses is situated to compensate for the phase shift of the lenses in the from arm. After combination of the two split parts by another $45^{\circ}$-grid and interference at the analyzer, the radiation is detected. In a reference measurement without sample, the mirrors are adjusted for identical optical lengths of both arms by checking for the first-order minimum of interference. The frequency-dependent phase changes are compensated automatically by a step-motor moving the mirror in the reference arm. After repeating the measurement with the sample, the frequency dependent phase shift caused by the sample can be calculated from the monitored mirror movements.

From transmission and phase shift the complex permittivity can be calculated by formulæ for optical multilayer interference [18. Here the thickness and optical parameters of the windows have to be taken into account, which can be determined by the measurement of the empty sample cell. The minimum value of $\varepsilon^{\prime \prime}$ detectable with this spectrometer is only limited by the maximum thickness of the sample that can be used. In our group values down to $\varepsilon^{\prime \prime}=10^{-2}$ have been measured.

\subsection{Fourier-transform spectrometer}

In the frequency region of $400 \mathrm{GHz}-1500 \mathrm{THz}$ commercially available Fourier-transform spectrometers (Bruker $113 v$ and $66 v / S$ ) are employed. Numerous sources, beam-splitters and detectors are used to measure transmission and reflection spectra. Up to now in the investigation of glass formers we reached frequencies up to $5 \mathrm{THz}$, which is the upper limit when using quartz plates as sample-cell 


\section{U. SCHNEIDER et al.}
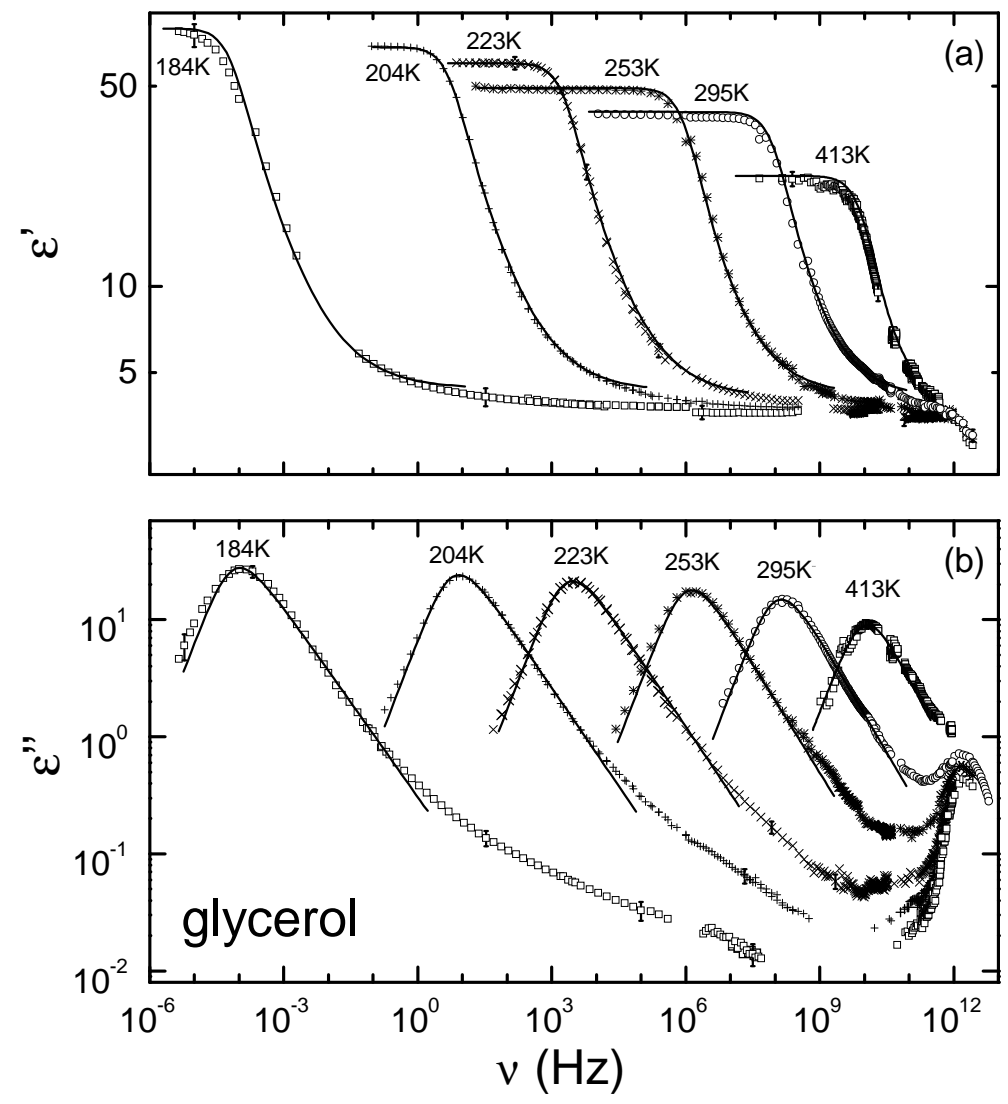

FIGURE 5: Combined spectra of glycerol for $\varepsilon^{\prime}(\mathrm{a})$ and $\varepsilon^{\prime \prime}(\mathrm{b})$ for various temperatures [10]. The lines are fits with the Cole-Davidson function [10].

windows. If patterns of standing waves are observed in the spectra one can unequivocally determine both the real and the imaginary part of the complex permittivity [18]. Otherwise the measured spectra are analyzed by a Kramers-Kronig transformation to obtain the complex permittivity.

\section{MEASUREMENT RESULTS}

In Figure 5 we present the combined results of all measurement techniques for the prototypical glass former glycerol [10]. The curves reveal the typical behavior of glass formers: A peak in $\varepsilon^{\prime \prime}$ shows up, that reflects the well known structural $\alpha$-relaxation which exhibits a dramatic change of time-scale with temperature. The $\alpha$-relaxation 


\section{WIDE RANGE DIELECTRIC SPECTROSCOPY...}

is followed by a second power law on the high-frequency side of the $\alpha$-peak (the "excess wing"). In the $\mathrm{GHz}-\mathrm{THz}$ region, not accessed in earlier dielectric investigations, a shallow $\varepsilon^{\prime \prime}(\nu)$-minimum is observed, which is of high relevance for theoretical investigations of the glass transition [1]. Finally, near $1 \mathrm{THz}$ the boson-peak shows up, well known from neutron and light scattering experiments [4 6]. For a detailed theoretical analysis of the present data, the reader is referred to [7, 8, 12].

\section{$4 \quad$ SUMMARY}

The large variety of dielectric techniques employed in our laboratory was described, laying special emphasis on the non-standard highfrequency methods. By combining these techniques, the collection of nearly continuous dielectric spectra covering about 20 decades of frequency has become possible. In this way, for the first time the temperature evolution of the large variety of dynamic processes present in glass-forming materials can be detected by a single experimental technique. As an example, spectra on the prototypical glass-forming liquid glycerol extending over a frequency range of more than 18 decades, well into the $\mathrm{THz}$ region, were presented.

\section{ACKNOWLEDGEMENTS}

We gratefully acknowledge the help of Yu.G. Goncharov and B.P. Gorshunov in setting up the quasi-optical spectrometer. We thank M. Dressel for assistance in some of the quasi-optical measurements and Th. Wiedenmann for technical support. This work was supported by the DFG Grant LO264/8-1 and partly by the BMBF, contract $13 \mathrm{~N} 6917$.

\section{$\underline{\text { References }}$}

[1] W. Götze and L. Sjögren, Rep. Progr. Phys 55, 241 (1992).

[2] K.L. Ngai, Comments Solid State Phys. 9, 127 (1979); D. Kivelson, S.A. Kivelson, X-L. Zhao, Z. Nussinov, and G. Tarjus, Physica A 219, 27 (1995); P. K. Dixon, L. Wu, S.R. Nagel, B.D. Williams, and J.P. Carini, Phys. Rev. Lett. 65, 1108 (1990). 


\section{U. SCHNEIDER et al.}

[3] A. Schönhals, F. Kremer, and E. Schlosser, Phys. Rev. Lett. 67, 999 (1991); A. Schönhals, F. Kremer, A. Hofmann, E.W. Fischer, E. Schlosser, Phys. Rev. Lett. 70, 3459 (1993); N. Menon and S.R. Nagel, Phys. Rev. Lett. 74, 1230 (1995).

[4] W. Petry and J. Wuttke, Transp. Theory Statist. Phys. 24, 1075 (1995).

[5] H.Z. Cummins, G. Li, Y.H. Hwang, G.Q. Shen, W.M. Du, J. Hernandez, and N.J. Tao, Z. Phys. B 103, 501 (1997).

[6] J. Wuttke, J. Hernandez, G. Li, G. Coddens, H.Z. Cummins, F. Fujara, W. Petry, and H. Sillescu, Phys. Rev. Lett. 72, 3052 (1994); W.M. Du, G. Li, H.Z. Cummins, M. Fuchs, J. Toulouse, and L.A. Knauss, Phys. Rev. E 49, 2192 (1994).

[7] P. Lunkenheimer, A. Pimenov, M. Dressel, Yu.G. Goncharov, R. Böhmer, and A. Loidl, Phys. Rev. Lett. 77, 318 (1996).

[8] P. Lunkenheimer, A. Pimenov, M. Dressel, B. Gorshunov, U. Schneider, B. Schiener, and A. Loidl, in: Supercooled Liquids: Advances and Novel Applications, ed. J.T. Fourkas, D. Kivelson, U. Mohanty, and K.A. Nelson (ACS Publications, Washington, DC, 1997) p. 168.

[9] P. Lunkenheimer, A. Pimenov, and A. Loidl, Phys. Rev. Lett. 78, 2995 (1997).

[10] U. Schneider, P. Lunkenheimer, R. Brand, and A. Loidl, J. Non-Cryst. Solids 235-237, 173, (1998).

[11] U. Schneider, P. Lunkenheimer, R. Brand, and A. Loidl, Phys. Rev. E 59, 6924 (1999).

[12] P. Lunkenheimer, U. Schneider, R. Brand, and A. Loidl, Contemp. Phys. (1999), in print.

[13] R. Böhmer, B. Schiener, J. Hemberger, and R.V. Chamberlin, Z. Phys. B 99, 91 (1995).

[14] R. Böhmer, M. Maglione, P. Lunkenheimer, and A. Loidl, J. Appl. Phys. 65, 901 (1989).

[15] Hewlett-Packard Product Note 8510-3 (1985).

[16] A.A. Volkov, Yu.G. Goncharov, G.V. Kozlov, S.P. Lebedev, and A.M. Prokhorov, Infrared Phys. 25, 369 (1985).

[17] A.A. Volkov, G.V. Kozlov, S.P. Lebedev, and A.M. Prokhorov, Infrared Phys. 29, 747 (1989).

[18] M. Born and E. Wolf, Priciples of Optics (Pergamon Press, Oxford, 1980). 